\title{
Resenha
}

\section{A revolução digital na saúde: como a inteligência artificial e a internet das coisas tornam o cuidado mais humano, eficiente e sustentável}

\section{Review}

The digital health revolution: how artificial intelligence and the internet of things make care more humane, efficient and sustainable

\section{Reseña}

La revolución de la salud digital: cómo la inteligencia artificial y el internet de las cosas hacen que la atención sea más humana, eficiente y sostenible

Luiz Pinto de Paula Filho ${ }^{1,2}$ Marcelo Lamy ${ }^{3}$

O livro A revolução digital na saúde: como a inteligência artificial e a internet das coisas tornam o cuidado mais humano, eficiente e sustentável procura demonstrar como a profusa revolução digital que ocorre atualmente - e iniciada com a criação do primeiro computador do mundo e a primeira linguagem baseada em algoritmos, em 1941 (1) - pode contribuir para a melhora da experiência do paciente nas ações de cuidado, promover a saúde e reduzir o custo dos serviços (1). A proposta principal dos autores é demonstrar como a tecnologia deve ser pensada e aplicada a serviço do melhor relacionamento do profissional da saúde com o paciente, contribuindo à humanização da saúde (1).

A experiência acadêmica e profissional dos autores - Claudio Lottenberg é mestre e doutor pela Escola Paulista de Medicina da Unifesp e foi presidente do Conselho Deliberativo do Hospital Israelita Albert Einstein; Patrícia Ellen da Silva é mestre em Administração Pública pela Universidade de Harvard (USA) e a atual secretária de Desenvolvimento Econômico, Ciência, Tecnologia e Trabalho do Estado de São Paulo; e Sidney Klajner é mestre pela Faculdade de Medicina da USP e presidente da Sociedade Beneficente Israelita Brasileira Albert Einstein - é profícua e contribui para demonstrar como a atual revolução digital pode contribuir à melhora da experiência dos atores atuantes no campo da saúde

\footnotetext{
1 Mestrando em Direito da Saúde, Universidade Santa Cecília (Unisanta), Santos, São Paulo, Brasil; advogado. https://orcid.org/0000-0002-1086-1512. E-mail: depaulalaw@uol.com.br.

${ }^{2}$ O presente trabalho foi realizado com apoio da Coordenação de Aperfeiçoamento de Pessoal de Nível Superior - Brasil (Capes) - Código de Financiamento 001.

${ }^{3}$ Doutor em Direito Constitucional, Pontifícia Universidade Católica (PUC), São Paulo, SP, Brasil; professor permanente e vice-coordenador, Programa de Pós-Graduação em Direito da Saúde, Universidade Santa Cecília (Unisanta), Santos, São Paulo, Brasil. http://orcid.org/0000-0001-8519-2280. E-mail: marcelolamy@unisanta.br.
} 
pública e privada, através do acréscimo de tecnologia às atividades médicas, ex. gr., visualizar todo o histórico de consultas e tratamentos de um paciente através de um prontuário eletrônico de saúde (electronic health records [EHR, sigla em inglês]), entre outros benefícios (1).

Observam os autores na Introdução da obra que há um novo paradigma da saúde em curso que é parte de uma transformação global, definida por Klaus Schwab como a quarta Revolução Industrial (2). Através do uso de inteligência artificial (IA), análise massiva de dados (big data), uso de internet das coisas (internet of things [loT, sigla em inglês]), entre outros, há um movimento em andamento que está mudando a forma como se pratica a medicina, onde se dará mais atenção à saúde, e não à doença, assim como o paciente será colocado no centro do sistema de saúde, e não mais o médico (2).

Nesse novo paradigma de saúde, onde a tecnologia é inserida como instrumento de auxílio a médicos, pacientes e gestores, os dados de um enfermo não são mais inscritos em papel e enviados ao arquivo pessoal do médico, mas inseridos num prontuário eletrônico unificado, onde são compartilhados com outros profissionais em tempo real, permitindo uma análise preditiva da situação da pessoa em consulta; cidadãos que precisam de atendimento de urgência poderão ser orientados com maior precisão por serviços que consideram seu perfil de saúde e encaminhados aos centros de saúde mais próximos, por terem seus dados on-line; os novos profissionais precisarão conhecer e compreender como a IA funciona e os auxilia na realização de suas atividades, exigindo uma remodelagem das escolas de medicina e dos métodos de ensino até aqui adotados; a remuneração de médicos e enfermeiros terá de ser reavaliada, para considerar as novas práticas que estão se desenhando (2).

Todos estes pontos não procuram fazer substituir os profissionais de saúde pela tecnologia, "[...] mas de fazer dela um instrumento real de eficiência naquilo que é o propósito máximo da medicina: tratar o paciente na sua integralidade, com qualidade, equidade e de maneira sustentável [...]", de modo que os instrumentos tecnológicos não sejam utilizados com o único propósito de cortar custos e gerar lucro, mas sim "[...] integrar os avanços digitais a um processo mais abrangente de acesso aos recursos da medicina e promoção da saúde $[\ldots] "(2)$.

Estabelecido o novo paradigma que se desenha na saúde, o Capítulo 1 da obra se dedica a uma breve história da revolução digital (Parte 1), demonstrando como a evolução 
humana está conectada com a evolução tecnológica e como o ser humano sempre mostrou interesse em desmembrar a inteligência humana, olhar seus componentes e os atribuir às máquinas, colocando-as a nosso serviço (3).

O século 20 foi marcado por descobertas importantes em todas as áreas das ciências, com destaque para os campos da física, matemática e de conhecimento do funcionamento do cérebro humano, que permitiram o desenvolvimento do conceito de IA e de máquinas baseadas nessa inovação (3). Desde a criação do primeiro computador no mundo e a primeira linguagem baseada em algoritmos, em 1941, até o estabelecimento e sedimentação do conceito de IA em 1956, da utilização do conceito de machine learning pela primeira vez, em 1959, até a explosão da internet em 1990, o avanço da tecnologia permitiu, a partir do fim do século 20 e início do século 21, que a IA descobrisse todo seu potencial e passasse a ser utilizada em diversos campos do conhecimento, sendo a saúde um dos campos mais beneficiados, com sistemas capazes de fazer diagnósticos, indicar tratamentos, operar equipamentos e realizar atividades que propiciam benefícios aos atores da saúde, como a medicina nunca havia visto (3).

Na Parte 2 do Capítulo 1, é abordado o impacto da tecnologia digital na saúde, desde a primeira Revolução Industrial (entre 1760 e 1840), passando pelas segunda (final do século 19) e terceira Revolução Industrial (a partir dos anos 1960), até chegar à atual quarta Revolução Industrial (iniciada na virada do milênio), que é marcada pela IA, a disseminação da robótica, a loT, veículos autônomos, impressão em 3D, entre outros. Os autores ressaltam que as empresas mais valorizadas atualmente têm seus negócios centrados nesse mundo virtual. $O$ avanço da tecnologia contribuiu para um aumento da capacidade de processamento de dados e uma diminuição dos custos desse processamento e, como isso, auxilia o desenvolvimento da IA, da loT, da criação do prontuário eletrônico de saúde e da telemedicina. Explicando cada um desses elementos, os autores demonstram como sua aplicação na saúde vem promovendo uma mudança de paradigma e contribuem para a implementação de políticas de prevenção e acompanhamento de morbidades.

No Capítulo 2, é apresentada a experiência alienígena com a digitalização e inserção de tecnologias à saúde. Demonstrando como o uso de IA, IoT, big data entre outras ferramentas são desenvolvidas ou aplicadas em países como o Canadá, onde são destacados exemplos de sucesso do uso de tecnologia em saúde; como foram desenvolvidos celeiros de produção de tecnologia voltada à saúde em Singapura e Israel; a 
discussão a respeito da proteção de dados havida na Alemanha, e; como os Estados Unidos têm investido nos estudos para a produção de novas ferramentas e mecanismos voltados à saúde, contribuindo à otimização do tempo despendido com internações, diminuição dos custos para hospitais e gestores e maior satisfação dos pacientes.

Evidentemente nem tudo é perfeito nesses países. Apontam os escritores que, enquanto o Canadá é o país mais avançado no uso de tecnologias digitais em saúde, o mesmo não se pode dizer da Alemanha, onde a transformação digital não estaria chegando aos pacientes; em Singapura, a digitalização dos serviços de saúde segue lenta em comparação ao ecossistema de pesquisa e inovação; nos Estados Unidos, as dificuldades para o acesso aos cuidados de saúde e a digitalização desse setor segue vagarosa, sendo superada pelas indústrias da comunicação, serviços profissionais, financeiros e seguros, comércio, governo, etc; e, em Israel, há complexa desigualdade no acesso aos serviços de saúde e aos resultados do sistema, bem como israelenses pobres não-judeus e aqueles que vivem na periferia das regiões norte e sul experimentam piores condições de saúde (4).

Para confirmar as informações demonstradas, são apresentados dados recentes sobre os investimentos realizados em tecnologia da saúde em cada país mencionado e como esse investimento tem gerado diminuição de custos; otimização do tempo; satisfação entre médicos e pacientes; e retornos científicos e financeiros como indicações ao prêmio Nobel e geração de patentes com royalties altíssimos (4), comprovando que investimentos em tecnologias digitais destinadas à saúde podem gerar benefícios não apenas à medicina e seus atores, mas também à economia dos países que investem nessa área.

No Capítulo 3, são verificados os grandes desafios que a saúde global tem experimentado, como o envelhecimento da população mundial, aumento da demanda e incidência de doenças crônicas, epidemias de obesidade e diabetes, custos da incorporação tecnológica, inflação médica e inadequação das formas de remuneração e de pagamento, etc, e como as inovações digitais apresentam soluções criativas e promissoras para a superação desses desafios (5).

Realçam-se exemplos como a integração de registros eletrônicos de mais de 24 mil pacientes em Ontário, no Canadá, que permitiram a identificação de três enfermidades como a principal causa de internações hospitalares; no Reino Unido, onde o diabetes e suas complicações são causas de hospitalização, o uso de um algoritmo permite identificar pessoas em alto risco e as encaminhar para um programa de mudança de comportamento; 
no noroeste de Londres, o programa Whole System Integrates Care (WSIC) aplica a tecnologia analytics sobre informações integradas das áreas de saúde e assistência social para identificar pessoas nos arredores em situação de maior fragilidade ou mais chances de internação e enviar uma equipe multidisciplinar para dar apoio a essas pessoas. Esses e outros exemplos reforçam como a tecnologia digital contribui para que crescentes desafios de saúde possam ser superados através do seu uso.

O Capítulo 4 reflete sobre o panorama brasileiro no horizonte da tecnologia digital em saúde. Inicia-se com um diagnóstico preocupante e temerário: "Hoje, o Brasil envelhece como a Suécia, morre como a Síria e adoece como a África do Sul, porque ainda somos um país com profundos contrastes." (6) Baseia-se em dados como o aumento do número de novos casos de diabetes, crescimento das enfermidades causadas por doenças infectocontagiosas (acentuado pelo baixo número de brasileiros que tem acesso à coleta de esgoto), crescimento da expectativa de vida, profunda desigualdade na distribuição de renda entre brasileiros e baixo investimento estatal em saúde ( $9 \%$ do PIB), constatando que, embora exista grande espaço para o crescimento do mercado de tecnologia em saúde no Brasil, a busca por maior eficiência do sistema é uma das mais sérias batalhas a serem travadas no país, estimando-se que o custo das ineficiências da saúde brasileira alcance anualmente cerca de US $\$ 18$ bilhões.

Embora existam iniciativas bem-sucedidas pela iniciativa privada, considerando-se a experiência de adoção do prontuário eletrônico (espécie de marco zero para dar início ao uso dos dados), até agora, tanto no setor público quanto no privado, os prontuários foram colocados em prática por pequeno número de instituições, assim como poucos hospitais nacionais alcançaram patamar elevado na implantação e uso dos novos recursos tecnológicos, demonstrando como a concretização da digitalização pelo sistema de saúde brasileiro ainda está longe de ser efetivada. Nem mesmo o anúncio, em 2017, de uma estratégia governamental para digitalizar todas as unidades básicas de saúde (UBS) foi suficiente para concretizar a implementação da digitalização da saúde no país - no momento do anúncio do projeto, dados do Ministério da Saúde (MS) indicavam que o prontuário eletrônico do paciente estava implementado em 15.488 das 42.818 UBS contabilizadas à época (6). Por outro lado, há boas notícias quanto à telemedicina, como a queda da mortalidade na cidade de Floriano $(\mathrm{PI})$ e a melhoria no atendimento de pacientes na cidade de Caruaru (PE), que conta apenas com médicos generalistas e, com o uso da telemedicina, 
passaram a receber suporte de médicos especialistas à distância. Aliás, as teleconsultas são a forma de telemedicina com maior potencial de expansão e, embora ainda não sejam liberadas pelo Conselho Federal de Medicina (CFM), há grande margem de crescimento quando for permitida (6). Com a entrada em vigor da Lei nำ13.989, de 15 de abril de 2020 um ano após a finalização do livro resenhado, em abril de 2019 (6) -, que autoriza o uso da telemedicina durante a crise ocasionada pelo coronavírus (art. 1ํㅡ, Lei 13.989/2020) (7), verifica-se que sua autorização decorre de situação excepcional e transitória cuja superação recolocará o uso da telemedicina à situação pretérita, de necessidade de liberação pelo Conselho Federal de Medicina. Que a situação transitória decorrente da atual pandemia possibilite que sejam realçados os benefícios que a telemedicina oferece, de maneira que seu uso seja devidamente regulado e liberado.

O Capítulo 5 é dedicado à formação médica na era digital. Como ressaltam os autores, as transformações proporcionadas pela IA só serão consolidadas se forem acompanhadas por profundas mudanças culturais, sendo fundamental uma mudança na estrutura do curso de medicina, para que os futuros profissionais tenham contato desde os primeiros semestres do curso com os princípios da IA e também com recursos tecnológicos: ensino centrado no aluno, com análise da capacidade de desenvolvimento de habilidades e de capacidades profissionais de cada estudante individualmente; formação de pequenos grupos de alunos para estudar, apresentar soluções de casos e exposição de decisões aos pacientes, avaliando-se, entre outras capacidades, a socialização do aluno com o paciente, eis que hoje a relação médico-paciente é cada vez mais horizontal e menos vertical, como ocorria antigamente. Nesse novo modelo de ensino e aprendizado, a ideia é que o profissional compreenda o objetivo de manter a saúde e não somente tratar doenças, diminuindo custos para hospitais e aumentado a satisfação dos pacientes (8).

Há escolas que já trocaram o método de ensino baseado no problem based learning, que privilegia a busca individual de solução, pelo team based learning, que envolve uma transformação de comportamento, ao ensinar os estudantes a trabalharem em equipe, quebrando o paradigma de um profissional que originalmente sempre trabalhou isolado, para um profissional que trabalha em conjunto com uma equipe multidisciplinar, todos pensando sobre o tratamento ideal para determinada condição médica (8).

O paciente no centro de tudo: o triple aim é o título do Capítulo 6, que destaca que a qualidade do sistema de saúde começou a ser fortemente discutida na virada do século nos 
Estados Unidos, após a publicação de dois relatórios pelo Institute of Medicine (IOM, sigla em inglês): Errar é humano: construindo um sistema de saúde mais seguro, nos anos de 1999; e Cruzando o abismo da qualidade: um novo sistema de saúde para o século 21, em 2001. Nos relatórios, ficou constatado haver um abismo entre a medicina ideal e a real, e se mostrou necessário haver toda uma mudança no sistema de saúde para se alcançar melhoria efetiva nos cuidados de saúde. Segundo os autores, o grande mérito desse debate foi pôr o paciente no centro do sistema de saúde pela primeira vez e mostrar que qualidade em saúde se traduz em cuidados adequados e, em última instância, em mortes evitáveis (9).

A partir dos debates engendrados por força dos referidos relatórios, surgiram conceitos estruturantes para o sistema de saúde que se propõem a solucionar o desafio da equação cuidados médicos versus custos crescentes, destacando-se o conceito de triple aim (meta tripla). Nesse conceito, o equilíbrio do sistema de saúde está atrelado à busca de três objetivos: i) melhorar a experiência do paciente com o cuidado, o que envolve também qualidade e segurança; ii) promover a saúde das populações; e iii) reduzir desperdícios, chegando ao custo adequado. O conceito se concentra no tratamento de populações e subpopulações e é focado na promoção da saúde e não da doença. Os cuidados de saúde são organizados numa rede de prevenção e acompanhamento que depende do uso de muita tecnologia, especialmente big data e analytics, para traçar o perfil básico das diferentes populações atendidas e desenhar programas de acompanhamento de acordo com esses diversos perfis, agindo nos três pilares inerentes a esse processo: melhora da experiência do paciente, desfecho do tratamento e o resultado financeiro (9).

O Capítulo 7 é dedicado à ética e segurança dos dados em saúde, travando a discussão sobre quem é o dono dos dados coletados, o direito à privacidade, a relação entre médico e paciente, o uso de algoritmos para a definição do tratamento a ser adotado, etc. As discussões éticas na área médica são intensas e muitos dilemas estão em debate, pois como atribuir valor ou um número a sensações humanas como dor, com base em critério como custos e expectativa de resultados? Como explicar ao paciente a lógica de uma máquina - o chamado dilema da caixa preta - e fazê-lo aceitar um diagnóstico ou tratamento indicado por sistemas? De quem é a responsabilidade em casos nos quais a máquina aponta uma conclusão errada? Essas questões ainda carecem de resposta.

Por outro lado, também a questão da segurança dos dados é essencial nesse debate, pois transações no mercado negro envolvendo informações de saúde são mais rentáveis 
hoje que informações obtidas junto ao cadastro de cartões de crédito, tendo o segmento de IA em saúde se tornado rapidamente um dos primeiros da lista dos mais atacados por hackers no mundo todo (10). No Brasil, o tema ganhou relevância e mereceu inovação legislativa com a promulgação da Lei no 13.709/2018 (11), que cuida da proteção de dados pessoais. Embora ainda não esteja totalmente em vigor, há um marco inicial por ela estabelecido de proteção de dados pessoais, definidos pela referida lei como sendo o nome e apelido, endereços residencial e eletrônico, número de documentos de identificação, dados de localização, endereço IP, testemunhos de conexão e dados obtidos por hospitais ou médicos que possibilitem a identificação da pessoa (10). Outrossim, os elementos essenciais resguardados pela Lei de Proteção de Dados são constituídos por direitos fundamentais e, em razão dos aspectos transnacionais do contexto de sua aplicação, por direitos humanos (12). Em que pese a regulamentação trazer maior proteção ao indivíduo que tem suas informações registradas quanto aos seus direitos humanos e fundamentais (10), já se observou, especificamente quanto ao uso do big data em saúde, que a atual Lei de Proteção de Dados oferece apenas algumas possibilidades, além do consentimento, para influenciar o destino dos dados pessoais, o que não é suficiente quando se considera a dinâmica dos grandes dados e implica a necessidade de se buscar novas formas de proteção em que esta funcionalidade seja efetiva e igualmente útil (12).

Finalmente, no Capítulo 8, são apresentados elementos para um plano diretor de digitalização da saúde, ressaltando-se que os países mais desenvolvidos já despertaram para a necessidade de uma política de Estado. No Brasil, ainda há carência de política e investimento públicos voltados à digitalização da saúde e nem mesmo o lançamento da Estratégia e-saúde para o Brasil, lançada em 2017 pelo MS e que reconheceu o registro eletrônico de saúde (RES) como ferramenta fundamental ao compartilhamento de informações, integração da atenção à saúde e suporte à decisão clínica, foi suficiente para a devida implementação de uma digitalização da saúde no Brasil (13).

$\mathrm{Na}$ Conclusão, os autores destacam estar em curso um encontro entre passado, presente e futuro na saúde, realçando que as mudanças promovidas pelas inovações digitais são sentidas, mais ou menos intensamente, em todos os campos da vida. Embora tais inovações estejam sendo aplicadas com profundidade na área da saúde, o quadro no Brasil adquire tons de cinza, pois carregamos uma tripla carga (triple aim) onde talvez a tecnologia 
"seja o único caminho para resolvermos os desafios da saúde que temos no país com a limitação e escassez de recursos financeiros" (14).

Sem perder de vista que o livro conta com glossário para ajudar a compreensão dos termos relativos à tecnologia e digitalização da saúde, a obra em questão é essencial para todos aqueles que se dedicam ao estudo da aplicação de tecnologias digitais e a digitalização da área da saúde, iluminando o debate sobre as inovações digitais e sua aplicação na saúde brasileira e contribuindo para o encontro de soluções que possibilitem uma real efetivação do direito fundamental à saúde em terras brasileiras.

\section{Referências}

1. Lottemberg $C$, Silva PE, Klajner $S$. A revolução digital na saúde: como a inteligência artificial e a internet das coisas tornam o cuidado mais humano, eficiente e sustentável. São Paulo: Editora dos Editores; 2019. 176 p.

2. Lottemberg C, Silva PE, Klajner S. A revolução digital na saúde: como a inteligência artificial e a internet das coisas tornam o cuidado mais humano, eficiente e sustentável. São Paulo: Editora dos Editores; 2019. Introdução: O novo paradigma da saúde.

3. Lottemberg C, Silva PE, Klajner S. A revolução digital na saúde: como a inteligência artificial e a internet das coisas tornam o cuidado mais humano, eficiente e sustentável. São Paulo: Editora dos Editores; 2019. Capítulo 1: Os caminhos da transformação.

4. Lottemberg $C$, Silva PE, Klajner S. A revolução digital na saúde: como a inteligência artificial e a internet das coisas tornam o cuidado mais humano, eficiente e sustentável. São Paulo: Editora dos Editores; 2019. Capítulo 2: Aexperiência em outros países.

5. Lottemberg $C$, Silva PE, Klajner S. A revolução digital na saúde: como a inteligência artificial e a internet das coisas tornam o cuidado mais humano, eficiente e sustentável. São Paulo: Editora dos Editores; 2019. Capítulo 3: Soluções inovadoras para grandes desafios.

6. Lottemberg $C$, Silva PE, Klajner S. A revolução digital na saúde: como a inteligência artificial e a internet das coisas tornam o cuidado mais humano, eficiente e sustentável. São Paulo: Editora dos Editores; 2019. Capítulo 4: O panorama brasileiro.

7. Brasil. Lei $n^{0} 13.989$, de 15 de abril de 2020. Dispõe sobre o uso da telemedicina durante a crise causada pelo coronavírus (SARS-CoV-2). Disponível em: http://www.planalto.gov.br/ccivil_03/_ato2019-2022/2020/Lei/L13989.htm. [Acesso em 21 jul. 2020].

8. Lottemberg C, Silva PE, Klajner S. A revolução digital na saúde: como a inteligência artificial e a internet das coisas tornam o cuidado mais humano, eficiente e sustentável. São Paulo: Editora dos Editores; 2019. Capítulo 5: A formação médica na era digital. 
9. Lottemberg C, Silva PE, Klajner S. A revolução digital na saúde: como a inteligência artificial e a internet das coisas tornam o cuidado mais humano, eficiente e sustentável. São Paulo: Editora dos Editores; 2019. Capítulo 6: O paciente no centro de tudo: o triple aim.

10. Lottemberg $C$, Silva PE, Klajner S. A revolução digital na saúde: como a inteligência artificial e a internet das coisas tornam o cuidado mais humano, eficiente e sustentável. São Paulo: Editora dos Editores; 2019. Capítulo 7: Ética e segurança de dados.

11. Brasil. Lei no 13.709, de 14 de agosto de 2018. Lei Geral de Proteção de Dados Pessoais (LGPD). Disponível em: http://www.planalto.gov.br/ccivil_03/_ato20152018/2018/lei/L13709.htm. [Acesso em 03.jul.2020].

12. Sarlet GBS, Molinaro CA. Questões tecnológicas, éticas e normativas da proteção de dados pessoais na área da saúde em um contexto de big data. Revista Brasileira de Direitos Fundamentais \& Justiça. 18 mar. 2020 [Acesso em 12 jul. 2020]; 13(41): 183-212. Disponível em: http://dfj.emnuvens.com.br/dfj/article/view/811.

13. Lottemberg $C$, Silva $P E$, Klajner $S$. A revolução digital na saúde: como a inteligência artificial e a internet das coisas tornam o cuidado mais humano, eficiente e sustentável. São Paulo: Editora dos Editores; 2019. Capítulo 8: Elementos para um plano diretor de digitalização da saúde.

14. Lottemberg C, Silva PE, Klajner S. A revolução digital na saúde: como a inteligência artificial e a internet das coisas tornam o cuidado mais humano, eficiente e sustentável. São Paulo: Editora dos Editores; 2019. Conclusão: O encontro entre passado, presente e futuro.

\section{Colaboradores}

Paula Filho LP contribuiu com redação da resenha. Lamy M contribuiu com a revisão crítica e aprovação da versão final.

\section{Como citar este artigo:}

Paula Filho LP, Lamy M. A revolução digital na saúde: como a inteligência artificial e a internet das coisas tornam o cuidado mais humano, eficiente e sustentável. Cadernos Ibero-Americanos de Direito Sanitário. 2020 jul./set.; 9(3): 225234.

http://dx.doi.org/10.17566/ciads.v9i3.707 